UC-34b

Issued: February 1977

\title{
MASTER
}

\section{Dynamical Zoning Within a Lagrangian Mesh by Use of DYN, a Stellar Pulsatiori Code}

by

John I. Castor *

Cecil G. Davis

David K. Davison* *

"Joint Institute for Laboratory Astrophysics, University of Colorado,

Boulder, Co 80302.

" C 3 Koshland Way, University of California, Santa Cruz, CA 95064.

\section{os Lamos}

scientific Iaboratory

of the University of Colifornio

los alAmOS, NEW MEXICO 87545

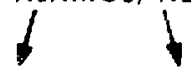

An Affirmotive Actior. / fqual Opportunity Emplayer 
Printed in the United States of America. Available from National Technical Information Service

U.S. Department of Commerce

$$
\begin{aligned}
& 5285 \text { Port Royal Rond } \\
& \text { Springtield, VA } 22161
\end{aligned}
$$

Price: Printed Copy $\$ 4.00$ Microfiche $\$ 3.00$

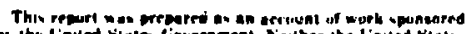

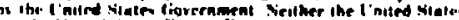

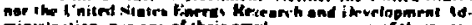
mintelrution. Mur ent of their emplavers. ner ont of theip con.

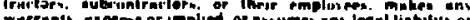

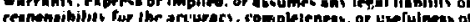

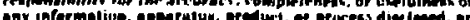

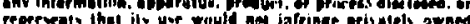
riehie. 
DYNAMICAL ZONING WITHIN A LAGRANGIAN MESH

3Y USE OF DYN, A STELLAR PULSATION CODE

by

John I. Castor

Cecil G. Davis

David K. Davison

\begin{abstract}
A method of dynamical zoning within a Lagrangian mesh is used to resolve the motion of the hydrogen ionization front in a timedependent nonlinear model of a pulsating star. The resulting coupling with the radiativetransfer improves the calculated light rurves. The method is described in some detail and then applied to a model of the Cepheid $n$ Aql.
\end{abstract}

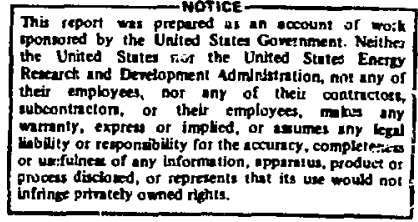




\section{INTRODUCTION}

The reliability of nonlinear stellar pulsation calculations has been questioned on the grounds of the zoning's infivence on light curve shapes. Keller and Mutschlecner ${ }^{1}$ showed that bumps on a certain Cepheid model's light curves are due to traversal of the hydrogen ionization front from one zone to another in the conventional Lagrangian mesh. The conventiona? Lagrangian technique, as applied to pulsating stars, requires that the ionization front move from zone to zone within the mesh. To resolve the front (maintain 3 or 4 zones within it), one must use a tremendcus number of zones in the star's outer regions.

The hydrogen ionization front motion is coupled with the radiative energy transfer, which can be treated with the equilibrium diffusion approximation. The front's motion may be determined from the Rankine-Hugoniot conservation conditions, provided the radiative flux con be found accurately. however, this requires that the ionization front be resoived. Clearly, the Lagrangian techrique is inadequate for solving stellar pulsation problems reliably if one must use a small number of zones in such problems oving to 1 imited computational resources. Similarly, we also deduce the inadequacy of the Eulerian techniques.

Here we discuss a hybrid, dynamical zoning iechnique that is neither Lagrangian, Eulerian, nor mixed Lagrangian-Eulerian, and which allows part of the computational mesh (calied the active region) to move with the ionization front in a pulsating star. Furthermore, the front is well enough resolved by this technique so that there can be no question about the accuracy of the radiative flux derived. Quantities near the ionization front change slowly in the coordinate system chosen for the active region. Thus we may use a fine mesh in this region and still use large time steps, provided our differencing technique does not need a Courant-like time-step control.

We describe below a computer code called oYN that uses an implicit differencing scheme in conjunction with the dynamic zoning technique just described. We conclude by demonstrating DYN's appiication to a model of the Cepheid $n \mathrm{AqT}$.

\section{NONLINEAR RADIAL STELLAR PULSATION EQUATIONS}

We assume spherical symmetry and adopt as a point of departure the Lagrangian system. The spatial coordinate is the mass within a distance $r$ of the center, $M_{r}$. Some of the dependent variables are the radial coordinate $r$, the flow velocity $u$, the specific volume $V$, pressure $P$, temperature $T$, internal 
energy $E$, and the radiative luminosity $L_{r}$ (the total ret power crossing a sphere of radius $r$ ). The conventional Lagrangian equations are then

$$
\begin{aligned}
& \left(\frac{\partial r}{\partial t}\right)_{M_{r}}=u, \\
& \left(\frac{\partial u}{\partial t}\right)_{M_{r}}=-\frac{G M_{r}}{r^{2}}-4 \pi r^{2}\left(\frac{\partial P}{\partial M_{r}}\right)_{t}, \\
& \left(\frac{\partial E}{\partial t}\right)_{M_{r}}+P\left(\frac{\partial V}{\partial t}\right)_{M_{r}}=-\left(\frac{\partial L_{r}}{\partial M_{r}}\right)_{t}, \\
& V=\left(\frac{\partial}{\partial M_{r}}\right)_{t}\left(\frac{A \pi}{3} r^{3}\right),
\end{aligned}
$$

and

$$
L_{r}=-\frac{\left(4 \pi r^{2}\right)^{2} 4 \sigma}{3 k}\left(\frac{\partial T^{4}}{\partial M_{r}}\right)_{t} \text {, }
$$

where $G$ is the gravitation constant, $\sigma$ is the Stefan-Boltzinann constant, and $\kappa$ is the Rosseland mean opacity.

Because we shall use zonal interfaces that are fixed not in mass, but in some other coordinate that we shall designate by $x$, a function of $M_{r}$ and $t$, we need to convert Eqs. (1)-(5) to the variables $x$ and $t$, rather than $M_{r}$ and $t$. We require the following identity, where $f$ is any physical variable.

$$
\left(\frac{\partial f}{\partial t}\right)_{M_{r}}=\left(\frac{\partial f}{\partial t}\right)_{x}-\left(\frac{\partial M_{r}}{\partial t}\right)_{x}\left(\frac{\partial f}{\partial M_{r}}\right)_{t} .
$$

(Recal1 that $\frac{\partial}{\partial t}[f(t, x)]_{x}=\frac{\partial}{\partial t}\left[f\left(t, M_{r}\right)\right]_{M_{r}}+\frac{\partial}{\partial M_{r}}\left[f\left(t, M_{r}\right)\right]_{t} \frac{\partial}{\partial t}\left[M_{r}(t, x)\right]_{x}$ when $M_{r}=M_{r}(t, x)$.) Combining Eq. (6) with Eqs. (1)-(3) gives

$$
\left(\frac{\partial r}{\partial t}\right)_{x}=u+\dot{M}\left(\frac{\partial r}{\partial M_{r}}\right)_{t},
$$




$$
\left(\frac{\partial u}{\partial t}\right)_{x}=-\frac{G M_{r}}{r^{2}}-4 \pi r^{2}\left(\frac{\partial P}{\partial \bar{M}_{r}}\right)_{t}+\dot{M}\left(\frac{\partial u}{\partial M_{r}}\right)_{t}
$$

and

$$
\left(\frac{\partial E}{\partial t}\right)_{x}+P\left(\frac{\partial V}{\partial t}\right)_{x}=\dot{M}\left[\left(\frac{\partial E}{\partial M_{r}}\right)_{t}+P\left(\frac{\partial V}{\partial M_{r}}\right)_{t}\right]-\left(\frac{\partial L_{r}}{\partial M_{r}}\right)_{t},
$$

where

$$
\dot{M} \equiv\left(\frac{\partial M_{r}}{\partial t}\right)_{x}
$$

Note that Eqs. (7)-(9) are valid regardless of the function of $M_{r}$ and $t$ that. may be chosen for $\dot{M}$. The precise procedure for choosing $\dot{M}$ is described in Sec. III, but, in short, the object is to minimize the right-hand sides of Eqs. (8) and (9). This makes the variables in a given zone, fixed in the $x$ coordinate, slow functions of time.

Equations (7)-(9) are converted to finite difference form to yield a fully implicit scheme. That is, the three basic variabies $r, u$, and $T$ are a!l specified at instants of time $t^{n}$ and a time step consists of the simultaneous solution for $r, u$, and $T$ at time $t^{n+1}$. The spatial zoning is indicated by a subscript, which is a half-odd integer for zonal quantities such as $P, T, V, E$, etc., and an integer for interface quantities such as $M_{r}, r$, $u, L_{r}$, etc. The index increases with radius. An implicit form must be used for Eqs. (7) and (8), as, otherwise, a Courant-type condition for stabiitity is imposed which is more stringent than the condition deriving from the motion of the front.

The finite-difference scheme that has been used most successfuliy is represented by the following equations:

$$
r_{i}^{n+1}-r_{i}^{n}=\frac{(\Delta t)^{n+1 / 2}}{2}\left(u_{i}^{n+1}+u_{i}^{n}\right)
$$




$$
+\frac{\dot{M}_{i}^{n+1 / 2}(\Delta t)^{n+1 / 2}}{4 D M_{i}^{n+1}}\left(r_{i+1}^{n+1}+r_{i+1}^{n}-r_{i-1}^{n+1}-r_{i-1}^{n}\right) \text {. }
$$

$$
\begin{aligned}
u_{i}^{n+1}-u_{i}^{n} & =-\frac{G M_{i}^{n+1 / 2}(\Delta t)^{n+1 / 2}}{r_{i}^{n+1} r_{i}^{n}} \\
& +\frac{2 \pi(\Delta t)^{n+\frac{1}{2}}}{3 D M_{i}^{n+1}}\left[\left(r_{i}^{n+1}\right)^{2}+r_{i}^{n+i} r_{i}^{n}+\left(r_{i}^{n}\right)^{2}\right] \\
& \times\left[(P+q)_{i-\frac{1}{2}}^{n+1}+(P+q)_{i-\frac{1}{2}}^{n}-(P+q)_{i+\frac{1}{2}}^{n+1}-(p+q)_{i+\frac{1}{2}}^{n}\right] \\
& \left.+\frac{\dot{M}_{i}^{n+\frac{1}{2}}(\Delta t)^{n+\frac{1}{2}}}{4 D M_{i}^{n+1}}\left(u_{i+1}^{n+1}+u_{i+1}^{n}-u_{i-1}^{n+1}-u_{i-1}^{n}\right)\right],
\end{aligned}
$$

and

$$
\begin{aligned}
& E_{i+1 / 2}^{n+1}-E_{i+1 / 2}^{n}+1 / 2\left[(p+q)_{i+1 / 2}^{n+1}+(p+q)_{i+1 / 2}^{n}\right] \\
& x\left(v_{i+\frac{1}{2}}^{n+1}-v_{i+\frac{1}{2}}^{n}\right) \\
& =\frac{L_{i}^{n+1}-L_{i+1}^{n+1}}{D M_{i+1 / 2}^{n+1}}(\Delta t)^{n+\frac{1}{2}} \\
& +1 / 2 \dot{M}_{i+\frac{1}{2}}^{n+\frac{1}{2}}(\Delta t)^{n+\frac{1}{2}}\left[\frac{E_{i+\frac{3}{2}}^{n+1}-E_{i+\frac{1}{2}}^{n+1}}{D M_{i+1}^{n+1}}+\frac{E_{i+\frac{1}{2}}^{n+1}-E_{i-\frac{1}{2}}^{n+1}}{D M_{i}^{n+1}}\right. \\
& \left.+(P+q)_{j+\frac{1}{2}}^{n+1}\left(\frac{v_{i+\frac{3}{2}}^{n+i}-v_{i+\frac{1}{2}}^{n+1}}{D M_{i+1}^{n+1}}+\frac{v_{i+\frac{1}{2}}^{n+1}-v_{i-\frac{1}{2}}^{n+1}}{D M_{i}^{n+1}}\right)\right] .
\end{aligned}
$$

5 
The boxes emphasize the changes to the basic finita-difference equations which result from the hybrid anproach. The auxiliary reiations are:

$$
\begin{aligned}
& v_{i+\frac{1}{2}}^{n+1}=\frac{4 \pi}{3} \frac{\left(r_{i+1}^{n+1}\right)^{3}-\left(r_{i}^{n+1}\right)^{3}}{D M_{i+1 / 2}^{n+1}} \\
& L_{i}^{n}=\frac{64 \pi^{2} \sigma}{3} \frac{\left(r_{i}^{n}\right)^{4}}{D M_{i}^{n}}\left[\frac{\left(T_{i-\frac{1}{2}}^{n}\right)^{4}}{k_{i-\frac{1}{2}}^{n}}+\frac{\left(T_{i+\frac{1}{2}}^{n}\right)^{4}}{k_{i+\frac{1}{2}}^{n}}\right] \\
& \times \frac{\left(T_{i-\frac{1}{2}}^{n}\right)^{4}-\left(T_{i+\frac{1}{2}}^{n}\right)^{4}}{\left(T_{i-\frac{1}{2}}^{n}\right)^{4}+\left(T_{i+\frac{1}{2}}^{n}\right)^{4}} \\
& q_{i+\frac{1}{2}}^{n}=\frac{C_{Q} F_{Q}^{n}}{v_{i+\frac{1}{2}}^{n}}\left[\operatorname{Max}\left(0, u_{i}^{n}-u_{i+1}^{n}\right)\right]^{2}, \\
& F_{Q}^{n}=\exp \left(1-\operatorname{Max}\left\{G_{Q} / \operatorname{Max}\left[\left(u_{i}^{n}-u_{i+l}^{n}\right) / \sqrt{P_{i+\frac{1}{2}}^{n} v_{i+\frac{1}{2}}^{n}}, G_{Q} / 100\right], 1\right),\right. \\
& O M_{i+\frac{1}{2}}^{n}=M_{i+1}^{n}-M_{i}^{n} \text {, } \\
& O M_{i}^{n}=1 / 2\left(D M_{i+\frac{1}{2}}^{n}+D M_{i-\frac{1}{2}}^{n}\right) \text {, } \\
& \dot{M}_{i}^{n+\frac{1}{2}}=\frac{M_{i}^{n+l}-M_{i}^{n}}{(\Delta t)^{n+\frac{1}{2}}} \text {, }
\end{aligned}
$$

and

$$
i_{i+\frac{1}{2}}^{n+\frac{1}{2}}=1 / 2\left(\dot{A}_{i}^{n+\frac{1}{2}}+\dot{M}_{i+1}^{n+1 / 2}\right) \text {. }
$$

The algorithm for evolving $\dot{M}_{i}^{n}$ is discussed in Sec. III. 
The factor $F_{Q}^{n}$ in trie artificial viscosity relation (16a), given by Eq. (16b), provides an exponential scaling to the artificial viscosity for the range $G_{Q} / 100 \leq \Delta u / a \leq G_{Q}$, where a is an approximation of the adiabatic sound speed and $G_{Q}$ is a user-controlled parameter. We have

$$
F_{Q}^{n}=\left\{\begin{array}{l}
1, G_{Q}<\frac{\Delta u}{a} \\
\exp \left(1-G_{Q} a / \Delta u\right), G_{Q} / 100 \leq \frac{\Delta u}{a} \leq G_{Q} \\
\exp (-99), \frac{\Delta u}{a}<\frac{G_{Q}}{100} .
\end{array}\right.
$$

This heuristic approach is a modification of a similar technique attributed tc $R$. Stellingwerf, which is used to prevent damping in the interior of the star.

Equations (11)-(13), into which the remaining equations have been substituted, may be regarded as a nonlinear system that determines $r_{i}^{n+1}, u_{i}^{n+1}$, and $T_{i+1}^{n+1}$. This system is solved, as usual, by use of the Newton-Raphson iechnique. The resulting linear system has a banded matrix whose band width is 11. It may be solved economically by the standard band matrix procedure.

The boundary conditions for the calculation are unchanged from those usually applied, as it is assumed that $\dot{M}$ vanishes in a region adjacent to each boundary.

A number of comments on the difference equations, (11)-(13), are in order. If the terms containing $\dot{M}$ are omitted, the equations become those introduced by Fraley, ${ }^{2}$ which yield an exact conservation law,

$$
\begin{aligned}
& {\left[\sum_{i} D M_{i}\left(\frac{\left(u_{j}\right)^{2}}{2}-\frac{G M}{r_{i}}\right)+\sum_{i} D M_{i+\frac{1}{2}} E_{i+1 / 2}\right]^{n+1} } \\
- & {\left[\sum_{i} D M_{i}\left(\frac{\left(u_{i}\right)^{2}}{2}-\frac{G M}{r_{i}}\right)+\sum_{i} D M_{i+\frac{1}{2}} E_{i+\frac{1}{2}}\right]^{n} } \\
= & \left(L_{i}^{n+1}-L_{N+1}^{n+1}\right)(\Delta t)^{n+\frac{1}{2}},
\end{aligned}
$$


when the usual boundary conditions are applied. $L_{i}$ and $L_{N+1}$ are the interior and exterior boundary luminosities, respectively.) Unfortunately, it does not seem possible to retain this feature if $\dot{M} \neq 0$. However, energy cunservation is more critical in the inner region of the model, where it is possible to set $\dot{M}=0$, so it seems desirable to use Fraley's equations as a basis.

It is also of interest to consider the extent to which the Rankine-Hugoniot conditions may apply across the ionization front for the finite-difference solution. We suppose that there is a solution of the difference equations which, for several zones near the front, is stationary in the sense that $T, V$, and $u$ are constant for a given zone or interface, and that $r_{i}^{n}=$ constant $_{i}+u_{F} t^{n}$ where $u_{F}$, a constant, is the front velocity. We further suppose that $\dot{M}$ is constant in space for those zones and that $4 \pi r^{2}$ can be considered constant where it multiplies spatial differences. Given these assumptions, Eqs. (11)-(13) yield

$$
\begin{aligned}
& u_{F}=u_{i}+\frac{\dot{M}}{4 \pi r^{2}}{ }^{\prime} v_{i}^{\prime}, \\
& 0=4 \pi r^{2}\left[(p+q)_{i-\frac{1}{2}}-(p+q)_{i+\frac{1}{2}}\right]+\dot{m} \frac{u_{i+1}-u_{i-1}}{2}, \\
& L_{i}-L_{i+1}+\dot{M}\left[{ }^{\prime} E_{i+1}{ }^{\prime}-{ }^{\prime} E_{i}^{\prime}+(p+q)_{i+\frac{1}{2}}\left(v^{\prime} v_{i+1}^{\prime}-{ }^{\prime} v_{i}^{\prime}\right)\right]=0,
\end{aligned}
$$

where

$$
v_{i}^{\prime}=\frac{D M_{i-\frac{1}{2}} v_{i-\frac{1}{2}}+D M_{i+\frac{1}{2}} v_{j+\frac{1}{2}}}{D M_{i-\frac{1}{2}}+D M_{i+\frac{1}{2}}}
$$

and

$$
{ }^{\prime} E_{i}{ }^{\prime}=\frac{O M_{i-\frac{1}{2}} E_{i-\frac{1}{2}}+O M_{i+\frac{1}{2}} E_{i+\frac{1}{2}}}{D M_{i-\frac{1}{2}}}+\frac{D M_{i+\frac{1}{2}}}{.}
$$

If we define

$$
v_{i} \equiv u_{i}-u_{i}
$$


we see that

$$
v_{i}=\frac{\dot{i n}}{4 \pi r^{2}}{ }^{\prime} v_{i}^{\prime}
$$

and

$$
(p+q)_{i+\frac{1}{2}}+\frac{\dot{m}}{4 \pi r^{2}} \cdot \frac{v_{i}+v_{i+1}}{2} \equiv K=\text { constant } .
$$

Inserting Eqs. (28) and (29) into Eq. (24) gives

$$
\begin{aligned}
& \left(L_{i}-\dot{M}{ }^{\prime} E_{i}^{\prime}\right)-\left(L_{i+1}-\dot{M}{ }^{\prime} E_{i+1}{ }^{\prime}\right) \\
& +\dot{H}\left[K-\frac{\dot{M}}{4 \pi r^{2}} \frac{v_{i}+v_{i+1}}{2}\right] \frac{4 \pi r^{2}}{\dot{M}}\left(v_{i+1}-v_{i}\right)=0,
\end{aligned}
$$

or

$$
L_{i}-\dot{M}\left({ }_{E}^{\prime}{ }_{i}^{\prime}+\frac{4 \pi r^{2}}{\dot{H}} \cdot K v_{i}-1 / 2\left(v_{i}\right)^{2}\right)=\text { constant, }
$$

or

$$
L_{i}-\dot{M}\left({ }^{\prime} E_{i}{ }^{\prime}+{ }^{\prime} P_{i}{ }^{\prime}{ }^{\prime} v_{i}{ }^{\prime}+1 / 2 v_{i}^{2}\right)=\text { constant, }
$$

where

$$
\begin{aligned}
& { }_{P_{i}}{ }^{\prime} \equiv K-\frac{\dot{M}}{4 \pi r^{2}} v_{i}=(P+q)_{i+\frac{1}{2}}+\frac{\dot{M}}{8 \pi r^{2}}\left(v_{i+1}-v_{i}\right) \\
& =(P+q)_{i-\frac{1}{2}}+\frac{\dot{M}}{8 \pi r^{2}}\left(v_{i-1}-v_{i}\right) .
\end{aligned}
$$


Therefore, we see that if the solution for $T, V$, and 1 yields smooth spatial variation of these quantities, so that $v_{i+1}-v_{i}, v_{i+1}-v_{i}, E_{i+\frac{1}{2}}-E_{i-\frac{1}{2}}$, and $q_{i+\frac{1}{2}}$ all tend to 0 away from the front, the appropriate conservation laws (Eqs. 28, 29, 32) are obeyed.

A final point about the difference equations is their stability. There is little point in checking stability against thermal disturbances, for the backward-differenced diffusion operator should guarantee stability. (Stability is confirmed by a simple analysis.) The more delicate stability question concerns density and velocity perturbations.

We examine the stability of Eqs. (11) and (12) by considering an analogous linear system with constant coefficients

$$
\begin{aligned}
& r_{i}^{n+1}-r_{i}^{n}=\frac{\Delta t}{2}\left[u_{i}^{n+1}+u_{i}^{n}+\frac{\dot{M}}{2 \Delta M}\left(r_{i+1}^{n+1}+r_{i+1}^{n}-r_{i-1}^{n+1}-r_{i-1}^{n}\right)\right] \\
& u_{i}^{n+1}-u_{i}^{n}=\frac{a^{2} \Delta t}{2(\Delta r)^{2}}\left(r_{i+1}^{n+1}-2 r_{i}^{n+1}+r_{i-1}^{n+1}+r_{i+1}^{n}-2 r_{i}^{n}+r_{i-1}^{n}\right) \\
& +\frac{\dot{M} \Delta t}{4 \Delta M}\left(u_{i+1}^{n+1}+u_{i+1}^{n}-u_{i-1}^{n+1}-u_{i-1}^{n}\right) .
\end{aligned}
$$

The eigenvalues $p$ of the amplification matrix (cf. Richtmyer and Morton ${ }^{3}$ ) are found by taking

$$
r_{j}^{n} \propto e^{i k j} p^{n}
$$

and likewise for $u_{j}^{n}$. We obtain

$$
p=\frac{1+i( \pm L \sin k / 2+\alpha \sin k)}{1-i\left( \pm L \sin \frac{k}{2}+\alpha \sin k\right)},
$$

where

$$
L=\frac{a \Delta t}{\Delta r} \text {, }
$$


and

$$
\alpha=\frac{i \Delta t}{2 \Delta M} \text {. }
$$

We see that $|p|=1$, so that the system is stable. If, in Eqs. (34) and (35), the $\dot{M}$ terms had been evaluated at $t^{n+1}$ rather than time centered, we would have found

$$
p=\frac{1 \pm i L \sin k / 2}{1-i(2 \alpha \sin k \pm L \sin k / 2)}
$$

which indicates instability if the front is subsonic. If all terms are evaluated at $t^{n+1}$, the amplification factors are

$$
p=\frac{1}{T-2 i \alpha \sin k \bar{F} 2 i L \sin k / 2}
$$

so that $|p| \leq 1$, giving stability. The very fact that $|p|<1$ in general for this modification indicates that the equations are dissipative in Richtmyer ard Morton's serise, although conservative. Use of such equations gave a large spurious damping which is avoided by use of Eqs. (11) and (12) as written.

\section{THE CHOICE OF aM/2t FOR DYNAMIC ZONING}

The routine that drives the motion of the mass interfaces is used for two principal purposes. The first and more basic is to try to keep a certain feature of the calculation stationary with respect to the mesh. The other is to ensure that the mesh remains relatively smooth in the meantime. Both objectives may be met by requiring the mass coordinate to satisfy an inhomogeneous diffusion equation in $x$ and $t$. That is,

$$
\left(\frac{\partial M}{\partial t}\right)_{X}=B \frac{\partial}{\partial x} \quad Y\left[\left(\frac{\partial M}{\partial x}\right)_{t}\right]+A
$$

In this equation $B, Y$, and $A$ are adjustable functions chosen to meet the above objectives best. 
So far the precise meaning of $x$ has not been stated. We may suppose that it is a continuo: $s$ variable that coincides with the zone index $i$ at its integer values; i.e., $x=1$ is the coordinate of interface $i$. The role of $A$ is that it be the desired value of $\partial M / \partial t$, neglecting the smoothing effect. If $A$ vanishes, the mesh relaxes with $\tau$ ime to a state in which $\Delta M \propto 1 / Y$. Therefore, $Y$ determines the steady-state zoning. The relaxation rate is fixed by $Y$ and $B$ together. Because it is the term $A$ that distorts the mesh and necessitates the diffusion term action, it is appropriate to take $B \propto\|A\|(\|A\|=\max |A|)$ to ensure that the mesh distortion is bounded regardless of the value of $A$. To make the diffusion term's smoothing effect uniform over the mesh, $B$ is also taken $\propto 1 / Y$. Therefore we assume that

$$
B=\frac{g\|A\|}{Y} \text {. }
$$

The constant $g$ remains to be determined. The best results have been obtained if the constant $\mathrm{g}$ was adjusted so that the time required for the smoothing effect to act over a distance of one zone, $1 / \mathrm{g}|\mathrm{A}|$, was about equal to the time that any part of the active region required to travel in mass an amount equal to the average zone mass $i$ in the active region, a time equal to $\Delta M /|A| \Delta i$. In other words, $g \simeq \Delta i / \Delta M$. Here $\Delta M$ is the mass, and $\Delta i$ is the number of zones, in the active region. The equation adopted is

$$
\frac{\partial M}{\partial t}=\frac{2\|A\| \Delta i}{\Delta M} \frac{\partial}{\partial x} Y\left(\frac{\partial M}{\partial x}\right)+A
$$

This is expressed in the finite-difference form

$$
\begin{aligned}
M_{i}^{n+1} & =M_{i}^{n}+\frac{4\|A\| \Delta i \Delta t^{n+\frac{1}{2}}}{\Delta M\left(Y_{i-\frac{1}{2}}^{n}+Y_{i+\frac{1}{2}}^{n}\right.}\left[Y_{i+\frac{1}{2}}^{n}\left(M_{i+1}^{n+1}-M_{i}^{n+1}\right)-Y_{i-\frac{1}{2}}^{n}\left(M_{i}^{n+1}-M_{i-1}^{n+1}\right)\right] \\
& +A_{i} \Delta t^{n+1 / 2},
\end{aligned}
$$

which is linear in the interface masses. 
The Function $A$

We wish to fix the value of $A$ so that the ionization front is stationary with respect to the mesh. We also may assume that $\dot{M}=A$ near the front. We showed in Sec. II that if $\dot{M}$ is chosen so that the front is stationary in the mesh, the jump conditions, Eqs. (28), (29), and (32), are obeyed. These relations can be solved for $\dot{M}$ in terms of flow quantities. Conversely, if $\dot{M}$ is determined from one of these relations, the front is stationary in the mesh. Any one of the three relations may be used for this purpose, but as they may not be exactly consistent, the reiation in which $M$ is fixed most sensitively is the best choice. This is the energy equation. It give:

$$
A_{C}=\frac{\Delta L}{\Delta\left(E+P V+\frac{1}{2} v^{2}\right)}
$$

where $A_{C}$ is taken to be the value of $A$ in the central part of the active region. The differences appearing here are taken between a zone close to $8000 \mathrm{~K}$ and one close to $15000 \mathrm{~K}$. The values of $v$ come from Eq. (27) where, from Eqs. 27$\rangle$ and (28),

$$
U_{F}=\frac{\Delta(u / v)}{\Delta(N / N)}
$$

The value $A_{C}$, givell by Eq. (46), is the value we use for $A$ in the center of the active region, where the ionization front is located. At the edges of the ragion, we use values that maintain a smooth transition to the Lagrangian mesh. Outside the active region, the mesh is uniform in the variable $\ln \left(M_{s}-M_{i}\right)$, where $M_{s}$ is the total mass of the star. Letting Deim be the increment in this variable, we set $\operatorname{Dmfac}=\exp ($ Deim) and define

$$
\begin{aligned}
& A_{L}=A_{i_{1}+1}=\left[\left(M_{s}-M_{i_{1}+1}\right) * \text { Dmfac }-\left(M_{s}-M_{i_{1}}\right)\right] i \Delta t, \\
& A_{U}=A_{i_{2}-1}=\left[\left(M_{s}-M_{i_{2}-1}\right)-\left(M_{s}-M_{i_{2}}\right) * \text { Dmfac }\right] / \Delta t,
\end{aligned}
$$

where the active region is delimited by the indices $i_{1}$ and $i_{2}$. 
If $A=A$ at the bottom edge of the active region, Eq. (44) tends to restore $\left(M_{s}-M_{i_{1}+1}\right)$ to the value $\left(M_{s}-M_{i}\right) /$ Dmfac. Similarly, Eq. (44) tends to restore $\left(M_{s}-M_{i_{2}-1}\right)$ to $\left(M_{s}-M_{i_{2}}\right) \star$ Dmfac when $A=A_{U}$ at the top edge; i.e., the increment in In $\left(M_{S}-H_{j}\right)$ just inside the active region is approximately Delm.

To combine the effects of the values $A_{C}, A_{L}$, and $A_{U}$, we define $A$ by

$$
A_{i}=\eta_{i} A_{E}+\left(1-\eta_{i}\right) A_{C} \text {, }
$$

where

$$
A_{E}=\left\{\begin{array}{l}
A_{L} \text { when } i \leq i_{0} \\
A_{U} \text { when } i>i_{0}
\end{array}\right\}
$$

( $i_{0}$ is the nominal coordinate of the ionization front), and

$$
\eta_{i}=\exp \left[\left(i-i_{1}-1\right) *\left(i-i_{2}+1\right) /\left(i_{2}-i_{1}+2\right)\right] .
$$

We note that $n_{\mathbf{i}_{1}+1}=n_{\mathbf{i}_{2}-1}=1$ and that $0<n_{\mathbf{i}_{0}} \ll 1$.

When we use Eq. (45) to calculate $A_{C}$ and subsequently obtain $A$ from Eq. (49) and 1 from the diffusion equation, Eq. $(44)$, we observe that the ionization front often drifts within the active region. To compensate for this drift, we correct $A_{C}$ with a feedback term. The ionization front is steepest near $10^{4} \mathrm{~K}$; thus the front's location may be assigned the value

$$
\langle i\rangle=i_{F}+\left(10^{4} K-T_{i_{F}}\right) /\left(T_{i_{F}+1}-T_{i_{F}}\right),
$$

where $i_{F} \leq\langle i\rangle \leq i_{F}+1$.

The deviation of $\langle i\rangle$ from the nominal coordinate $i_{0}$ is used to modify $A_{C}$.

$$
A_{C}=A_{C}-\varepsilon_{A}\left|A_{C}\right|\left(<i>-i_{0}\right),
$$


where $E_{A}$ is a small constant $(0.05-0.20)$. Equation (45) usually estimates the actual front motion to within \pm 5 or $10 \%$ so the front remains within one or two zones of its nominal positicn in the active region.

The nominal index $i_{0}$ of the ionization front is chosen approximately midway between the limiting indices $i_{1}$ and $i_{2}$. Equations (45) and (52) keep the ionization front location close to the index $i_{0}$. As the calculation proceeds, the front sweeps through the outer region of the star. This causes mass transfer from one end of the active region to the other, and the front approaches, in mass, one of the edges of the active region, as illustrated in Fig. 1.

In Fig. 1 we see both the effect of the mass motion through the mesh and the effects of Eqs. (47) and (48). The latter are demonstrated by the mesh smoothness, as a function of zone, at the edges of the active region. Figure 1 also shows the mesh fineness at the ionization front.

The ionization front is centered by index in the active region through the action of the feedback term in Eq. (52). To keep the front also centered in mass, we must periodically increment $i_{1}$ and $i_{2}$ by one unit.

The criterion we have found successful in determining the moment for incrementing $i_{1}$ and $i_{2}$ is

$$
\left|\ln \left(M_{s}-M_{i_{0}}\right)-\frac{1}{2}\left[\ln \left(M_{s}-M_{i_{1}}\right)+\ln \left(M_{s}-M_{i_{2}}\right)\right]\right|>\text { Deviatn*Delm, }
$$

where $M_{s}$ is the mass of the star, Delm is the (constant) increment in $\ln \left(M_{s}-M_{j}\right)$ for $i$ outside the active region, and Deviatn $>0.5$. We have found that Deviatn $=0.6$ provides good results. Note that, when $i_{1}$ and $i_{2}$ are incremented, the averäge $\frac{1}{2}_{2}\left[\ln \left(M_{s}-M_{i_{1}}\right)+\ln \left(M_{s}-M_{i_{2}}\right)\right]$ changes by a value approximateiy equal to Delm, but $\ln \left(M_{s}-M_{i_{0}}\right)$ changes by a value that is small compared to DeIm.

The value $A_{C}$ we have chosen for $A$ in the center of the active region serves to minimize the right-hand sides of Eqs. (8) and (9), as we have mentioned before. We imposed this condition to obtain solutions for $T, u$, and $V$ which vary slowly in time. We showed earlier that this allows us to conclude that the conservation laws are obeyed.

At the edges of the active region, the $\dot{M}$ values obtained from Eqs. (44) and $(47)-(49)$ are not subject to the same restrictions as those obtained at the 
center. At the center, we are concerned about steep gradients' effect on the solution as a whole. This problem does not arise at the edges of the active region.

The function $n_{i}$, defined by Eq. (50), is small in the center of the active region, approximately equal to 0.001 . If $\|A\|=\max |A|$ is large compared to $\left|A_{C}\right|$, the value of $A$ in the center of the active region, calculated from Eq. (49), may differ significantly from the required value $A_{C}$. Thus we impose the conditions

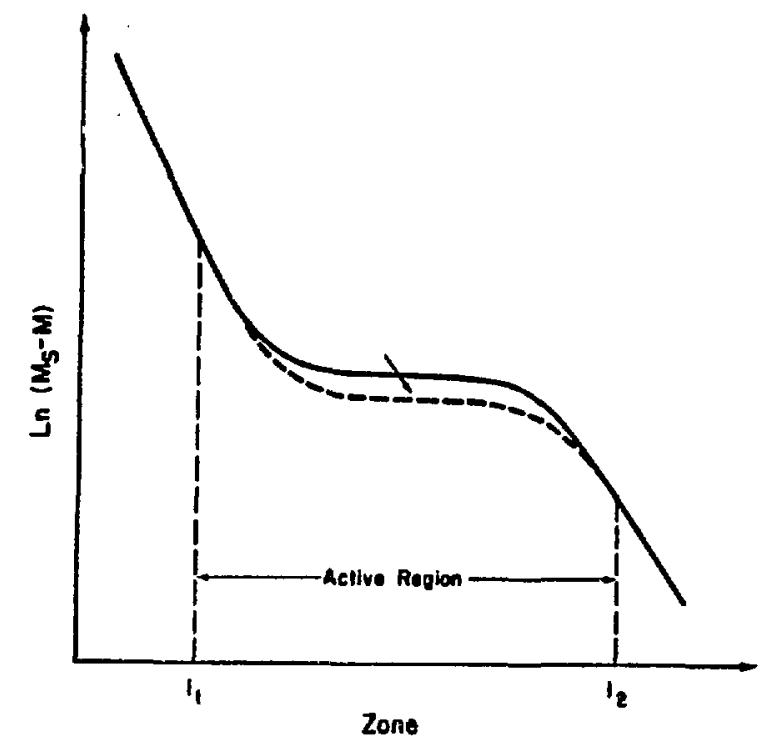

Fig. 1.

Ionization front motion.

$$
\left|A_{L}\right| \leq 10 \cdot\left|A_{C}\right| \text { when }\left|A_{L}\right|>|\bar{A}|
$$

ard

$$
\left|A_{U}\right| \leq 10 \cdot\left|A_{C}\right| \text { when }\left|A_{U}\right|>|\bar{A}| .
$$

If $|\bar{A}|=0,\|A\| \leq 10 \cdot\left|A_{C}\right|$.

The final adjustment we apply to $A$ is

$$
A_{i}=\left(1-n_{i}\right)\left[A_{i}-\left(A_{i_{0}}+A_{i_{0}+1}\right) / 2\right]+A_{i} \text {, }
$$

where $A_{C}$ is obtained from Eq. (52) and $A_{i_{0}}, A_{i_{0}}+1$, and $A_{i}$ result from Eq. (49). This adjustment allows us to correct the effect of $A_{E}$ on the central values of A. We obtain: $A_{i}^{-} \sim \eta_{i}^{2} A_{E}+\left(1-\eta_{i}^{2}\right) A_{C}^{-} \sim A_{C}^{-}$for $i \sim i_{0}$. 
To summarize, we write our method of obtaining $A$ as an algorithm.

1. Obtain $A_{c}$ as the central value of $A$ from the Rankine-Hugoniot relation, Eq. (45).

2. Obtain the edge values $A_{L}$ and $A_{U}$ by considering the mass changes in the edge cells required to maintain nominal size [Eqs. (47) and (48)].

3. Adjust $A_{C}$ by a feedback term to keep the ionization front centered in the active region [Eq. (52)].

4. Limit the size of $\left|R_{L}\right|$ and $\left|A_{C}\right|$ by means of Eq. (54).

5. Combine the adjusted values $A_{L}, A_{C}$, and $A_{U}$ by use of Eq. (49).

6. Correct the effects of the edge values on the central values with Eq. (55).

\section{The Function $Y$}

The hydrogen ionization front is a region of rapid spatial variation of temperature. Therefore, good front resolution is obtained if the relative temperature increment between adjacent zones is controlled.

We have seen that Eq. (41) tends to smooth out the mass zoning in the active region. It also controls the zone sizes in such a way that $\Delta M \sim 1 / Y$. This suggests that $Y$ should be a function that is large in the center of the active region (near the ionization front) and is small and approximately constant towards the edges of the active region. This latter requirement arises from a need to allow the values $A_{L} A_{U}$ to control the zoning at the edges by Eqs. (44) and (49). Furthermore, $Y$ should be a smooth function of $X$. Considering these requirements, we define

$$
\gamma_{i}^{n}=\left(\gamma_{\max }^{n}-1\right) \exp \left[-\left|c_{s}(i-\langle i\rangle)\right|^{\alpha}\right]+1
$$

where

$$
\begin{aligned}
Y_{\max }^{n} & =F_{s}^{n}\left(\exp \left\{\left[c_{s}\left(i_{2}-i_{1}\right) / 3\right]^{\alpha}\right\}+1\right), \\
F_{s}^{n} & =\text { Average }\left\{F_{s}^{n-1}, \exp \left[w_{s}-\left(x_{B}^{n}-x_{A}^{n}\right]\right) / F_{s}^{n-1}\right\}, \\
c_{s} & =2 / W_{s} .
\end{aligned}
$$


$W_{S}$ is the nominal width of the ionization front in the $x$ coordinate, and $x_{B}^{n}, x_{A}^{n}$ are the interpolated $x$-values where $T=8000$ and $15000 \mathrm{~K}$, respectiveiy. $<i>$ was defined previously as the interpolated $x$-value corresponding to $T=10000 \mathrm{~K}$. < $i>$ is also evaluated at time $n$, but we umit the superscript for the sake of notational clarity.

Some remarks are in order. First, when $F_{5}=1$ and $\left.|i-\langle i\rangle|\right\rangle\left(i_{2}-i_{1}\right) / 3$, $1<Y_{i}^{n}<2$. Furthermore, as $\mid i-\langle i>|$ increases (as $i$ moves farther from $\langle i>), Y_{i}^{n}$ decreases smoothly towards 1 . We see, then, that $Y_{i}^{n}$ is small and approximately constant towards the edges of the active region.

Next, we have $y_{\max }^{n}=y_{\langle i>}^{n}=F_{s}^{n}\left(\exp \left\{\left[c_{s}\left(i_{2}-i_{1}\right) / 3\right]^{\alpha}\right\}+1\right)$. As we choose to set $w_{s}=\left(i_{2}-i_{1}\right) / 4$ and $c_{s}=2 / w_{s}$, we obtain

$$
\left.y_{\max }^{n}=F_{s}^{n} \mid \exp \left[(i / 3)^{\alpha}\right]+:\right\} .
$$

If $F_{s}^{n} \sim 1$, we see that $Y_{\max }^{n}$ is a rapidly increasing function of $\alpha$. Thus, for $\alpha \sim 2$ and $F_{s}^{n} \sim 1, \gamma_{1}^{n}$ meets the requirements stated above (large in the vicinity of the ionization front and small and constant towards the edges of the active region).

$F_{S}^{n}$, defined by Eq. (58), is a feedback term. It adjusts the value of $\gamma_{\max }^{n}$ to maintain the relation $W_{S} \sim\left(x_{B}-x_{A}\right)$. In actual practice, we do not obtain $w_{S} \sim\left(x_{B}-x_{A}\right)$ for all $\alpha .\left(x_{B}-x_{A}\right)$ increases as $\alpha$ increases; i.e., the number of zones in the ionization front is controlled by $\alpha$. We have found that $w_{s}=\left(i_{2}-i_{1}\right) / 4=\left(x_{B}-x_{A}\right)$ for some $\alpha$ between 1 and 2 . We also have found that smaller values of $\alpha$ allow the code to run faster (with less resolution of the ionization fron $i$ ).

In developing. the feedback term $F_{s}^{n}$, we considered using $F_{S}^{n}=\exp \left[W_{S}-\left(x_{B}^{n}-x_{A}^{n}\right)\right]$. We rejected this choice, as it resulted in al ternateperiod oscillations in the value of $\left(x_{B}^{n}-x_{A}^{n}\right)$. Equation (58) is a variant of the Newton iteration on the relation $\left(F_{S}\right)^{2}-\exp \left[w_{S}-\left(x_{B}-x_{A}\right)\right]=0$. 
IV. GENERAL DYN CODE FEATURES

The DYN code incorporates the features described in Secs. II and III. It ailso includes a static-model-generating subroutine (Static), problem dump save and read subroutines (Save and Restor), a pressure-opacity rable-reading subroutine (Initial), a table traverser (EOS), and plotting routines (Ploter, Profils, etc). Figure 2 is a flowchart of the code.

Time Step Control

In subroutine Solve, the quantities $\delta_{3 j+1}=\Delta T / T, \delta_{3 j+2}: \Delta R / R$, and $\delta_{3 i+3}=\Delta u / a$ are calculated, where $a=\sqrt{P V}$ is approximately equal to the sound speed. From these, we get $|\delta|=\max \left|\delta_{j}\right|$, which may decrease the time step when $|\delta|$ takes on certain values. (See loop B-D in Fig. 2. On loop B-D, $\varepsilon<|\delta|<\varepsilon^{\prime}$ and $I_{S} \geq I_{S}(\max )$ or $|\delta|>\varepsilon^{\prime}$, where $\varepsilon \sim 10^{-6}, \varepsilon^{\prime} \sim 0.3$, and $I_{S}(\max ) \sim 6$. $\left(\varepsilon, \varepsilon^{\prime}\right.$, and $I_{s(\max )}$ are input parameters.)

In subroutine Accept, $\left|\delta_{T}\right|=\max |\Delta T / T|$ and $\left|\delta_{V}\right|=\max |\Delta V / V|$ are calculated. The time step is cut whenever $\left|\delta_{T}\right| / L_{T}>1$ or $\left|\delta_{V}\right| / L_{V}>1$ where $L_{T}$ and $L_{V}$ are input tolerances $\left(L_{T} \sim L_{V} \sim 0.1\right)$. It is increased when $\left|\delta_{T}\right| / L_{T}<0.5$, $\left|\delta_{V}\right| / L_{V}<0.5$, and $\Delta t / D t l i m<0.5$. Dtlim is an input parameter (Dtlim $\sim 1 .-5$. ). Static Model

The characieristics of the static model that the DYN code produces are controlled by the star mass, luminosity, and effective temperature paraneters. The zoning is controlled by the Dmfac parameter, an upper limit to the ratio $\left(M_{s}-M_{j}\right) /\left(M_{s}-M_{j+1}\right)$, where $M_{s}$ is the star mass. The outer zone temperature is obtained from $T^{4}=T_{e}^{4}(3 / 4)\left(q_{0}+\tau_{0}\right)$, where $q_{0}$ is the Hopf function, evaluated at $\tau_{0}$, the optical depth of the surface zone. (See Mihalas, ${ }^{4}, \mathrm{ch} .2$ ). $\tau_{0}$ and $q_{0}$ are input parameters.

Initial Velocity Distribution

The initial velocity distribution for the dynamic calculation is obtained from $u_{i}=u_{0}\left(R_{j} / R_{s}\right)^{6.3}$, where $R_{s}$ is the surface radius and $u_{0}$ is an input parameter.

\section{Mass Zoning Control}

The mass zoning is controlled by four input parameters. The coefficient $\varepsilon_{A}$ of the feedback term in Eq. (52) is the first of these. A nominal value for $\varepsilon_{A}$ is 0.1 . We have found that the code sometimes runs faster when small $E_{A}$ values are used $(0.05$ as opposed to 0.2$)$. This is understandable because the uncorrected value of $A_{C}$ is the value that minimizes the changes in the problem 


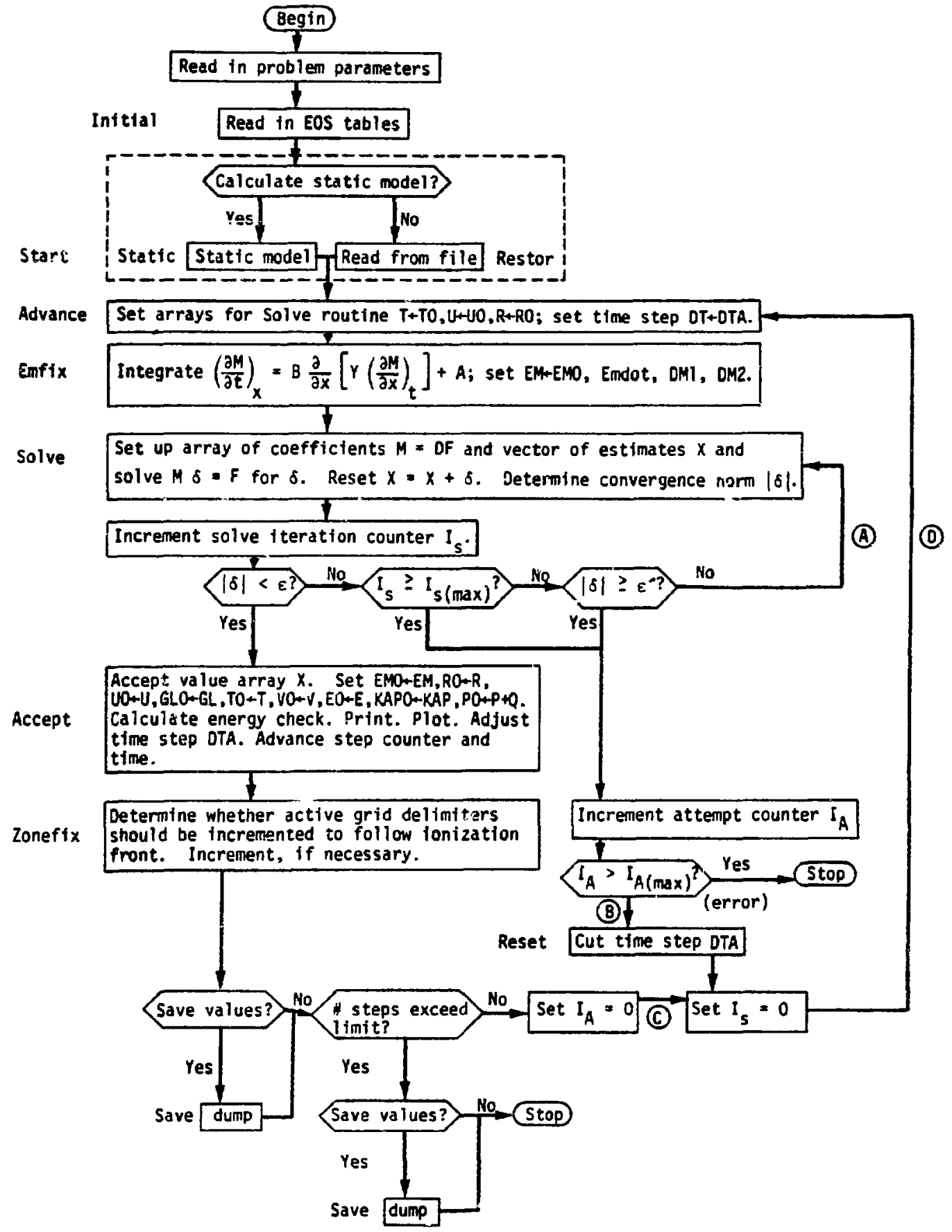

Fig. 2.

DYN program flowchart. :oop $A$ is an iteration on $R, U, T$ to obtain a small value of $|\delta|$. Loop $B$. D is an iteration on $\Delta t$. Loop C - D is an iteration on the problem step counter. 
variables, as we have seen. Some feedback is necessary, however, to line the center of the active region to the ionization front position.

The second zoning control parameter is the Deviatn factor in Eq. (53). This parameter controls the riming of the active region shift in the Lagrangian mesh which is necessary to keep the ionization fronit approximately centered, in mass, in the active region. A satisfactory Deviatn value is 0.6 .

The third parameter is the quantity $|\bar{A}|$ in Eq. (54). $|\bar{A}|$ is the floor value for $\left|A_{E}\right|=\max \left(\left|A_{L}\right|,\left|A_{U}\right|\right)$ below which the dominance of $\left|A_{E}\right|$, compared to $\left|A_{C}\right|$, is not controlled. When $\left|A_{E}\right|>|\bar{A}|, A_{L}$ and $A_{U}$ are adjusted so that $\left|A_{E}\right| \leq 10 \cdot\left|A_{C}\right|$.

The last zoning control parameter is the exponent $\alpha$ in Eq. (56). a controls the distribution of mass within the active region. Larger $\alpha$ values bring more zones into the ionization front, increasing its resolution. A suggested $\alpha$ value at low amplitudes is 1 . At higher amplitudes, it may be necessary to increase $\alpha$ to 1.5 or 2 to keep the velocity curves smooth.

Plotting Facilities

DYN has facilities for plotting se:eral parameters as functions of time, mass, and zone number. The plotting subroutines Ploter and Profils may be modified easily to provide other plots.

For convenience, we have associated a small integer with each plot.

\begin{tabular}{|c|c|c|}
\hline & Ordinate & Abscissa \\
\hline & Radius at $\tau=2 / 3$ and at $T=10^{4} \mathrm{~K}$ & Time \\
\hline & Velocity at $\tau=2 / 3$ and at $T=10^{4} \mathrm{~K}$ & Time \\
\hline & Luminosity at $\tau=2 / 3$ and at the surface & Time \\
\hline & Mass depth of ionization front (at $T=10^{4} \mathrm{~K}$ ) & Time \\
\hline & Kinetic energy (totar) & Time \\
\hline & Temperature & Mass \\
\hline & Velocity & Mass \\
\hline & Density & Mass \\
\hline & Ln (pressure) & Mass \\
\hline & Mass & Zone number \\
\hline & Opacity & Zone number \\
\hline & $\left(\frac{\partial \ln T}{\partial M}\right)_{t}=\frac{3 L_{s}}{16 \pi \sigma G_{s}}\left(\frac{k P}{T^{4}}\right)$ & Zone number \\
\hline & old temperature & 01d mass \\
\hline & old velocity & 01d mass \\
\hline
\end{tabular}




$\begin{array}{rll}\text { I } & \text { Ordinate } & \text { Abscissa } \\ 23 & \text { Old density } & \text { O1d mass } \\ 24 & \text { Ln (old pressure) } & \text { 01d mass } \\ 25 & \text { 01d mass } & \text { Zone number }\end{array}$

The plots indexed as 11-17 are intended primarily for diagnostic use and are accessed by a call to the Profils subroutine. Those indexed as 21 to 25 are no different from those indexed as 11 to 17 when Profils is called from subroutine Accept, as usually is the case

Plot selection is controlled by a single input integer. The integer's bit pattern, considered as a sequence of indexed bins, is examined for bits in the positions tabulated above. Thus, for example, the integer 14, with bit pattern 1110 conjures up the plots indexed as 1,2 , and 3 (the right-most bit has index 0$)$. By this arrangement, we are able to choose among 30 plots with a 10-digit integer.

Plot timing is controlled by input parameters, those indexed as 1 to 5 being treated separately from those indexed as 11 to 25 . Examples of the use of these parameters are given in Sec. $V$.

\section{SAMPLE PROBLEM}

We illustrate DYN code use by running an actual problem, an attempt to construct a model of $\eta \mathrm{Aql}$, a nearby galactic Cepheid for which there are ample observational data. From $\mathrm{Pel},{ }^{5}$ we get $\theta_{e}=0.898$, which corresponds to an effective temperature $T_{e}=5613 \mathrm{~K}$. We calculate a mean effective temperature of $5633 \mathrm{~K}$ from data in Whitney's Table $4,{ }^{6}$ and of $5659 \mathrm{~K}$ from data in Oke's Table $3 .^{7}$ Schmidt ${ }^{8}$ gives $5943 \mathrm{~K}$. Although the trend seems to be upward, except for Pel's result, we adopt $T_{e}=5610 \mathrm{~K}$ as a starting value. Christy ${ }^{9}$ describes a nonlinear model with $T_{e}=5600 \mathrm{~K}$, so we may be on familiar ground.

We derive the luminosity from the relation

$$
L_{T}=4 \pi \sigma R_{S}^{2} T_{e}^{4},
$$

where the radius $R_{S}$ is $4.75\left(10^{12}\right) \mathrm{cm}$, as reported by whitney. ${ }^{6}$ This differs from Christy's value of $R_{S}, 4.44\left(10^{12}\right) \mathrm{cm}$, but his model oscillates in the 
first harmonic mode (we anticipate oscillation in the fundamental mode). He get $L_{T}=1.59\left(10^{37}\right)$ ergs/s from Eq. (61).

Keeping $L_{T}$ and $T_{e}$ fixed, we guess the star mass $H_{S}$ using Eq. (5) by King, Cox, Eilers, and Davey. ${ }^{10}$

$$
\log \frac{L_{T}}{L_{0}}=3.48 \log \frac{M_{S}}{M_{0}}+0.68 \text {. }
$$

where $L_{0}$ and $M_{0}$ are the respective solar mass and luminosity. We get $M_{s}=1.38\left(10^{34}\right) \mathrm{g}$.

From the period mean density relation, Cox's $5 \mathrm{q} .(2.3), 11$ we derive

$$
\text { II } \sqrt{M_{S}} \sim K \text {, a constant, }
$$

for fixed $R_{s}$.

As we do not expect Eq. (62) to give us a model with the correct period on the first try, we use Eq. (63) in an iterative scheme to obtain a model with the correct period. To determine the pulsation period that corresponds to a given set of parameters $\left(T_{e}, L_{T}, M_{s}\right)$, we use a linear, noradiabatic model code and iterate on the mass. Our algorithm is as follows.

1. Accept $T_{e}$ and $R_{s}$ values given above.

2. Calculate $L_{T}$ using Eq. (61).

3. Calculate $M_{S}$ using Eq. (63).

4. Determine the period II that corresponds to $\left(T_{e}, L_{T}, M_{s}\right)$ using the linear code.

5. If $\pi$ is close enough to the period $\pi_{n}$ of $n$ Aql (7.18) days), stop; otherwise, proceed to 6 .

6. Calculate a new mass using Eq. (63).
a. $M_{s}=M_{s}$
b. $M_{s}=\left(\pi / \Pi_{n}\right)^{2} M_{s}$

7. Return to step 4 with the new mass value.

Using tinis algorithm, we get $M_{s}=1.84\left(10^{34}\right) \mathrm{g}$, which yields $I I=7.20$ days for the (pulsationally uristable) fundamental mode. 
For clarity, we repeat that the values we accept for DYH code starting parameters are $\left(T_{e}, L_{T}\right.$, and $\left.M_{s}\right)=\left(5610 \mathrm{~K}, 1.59\left(10^{37}\right)\right.$ ergs $/ \mathrm{s}$, and $1.84\left(10^{34}\right) \mathrm{g}$.

He are now ready to start the nonlinear calculation with the DYN code. The parameters $T_{e}, L_{T}, M_{S}$ are entered on card I (see Fig. 3 and Table I) of the data, in the proper units. Parameter $2 a$ is appropriate to the King $4 a$ mixture. Cara 4 indicates that we will run the problem for 2500 steps. Because parameter $5 \mathrm{~d}$ is TRUE, $4 \mathrm{~b}$ is 250 , and $4 \mathrm{f}$ is $2^{20}-1$ (first 19 bits of the PL.TCTRL parameter turned "on"), we will get at least $7(2500 / 250)=70$ plots (the diagnostic plots indexed as 11 to 17, at step intervals of 250). Thus, some provision must be made for microfilm or microfiche output.

Using the input cards shown in Fig. 3, we get a successful run that produces the partial listings shown in Figs. 4-7.

We continue the run by pumping for 4000 cycles (set parameter $5 \mathrm{c}$ to TRUE, parameter $2 \mathrm{~h}$ to 1.20$)$. Finally, we reset the pump parameter $5 \mathrm{c}$ to FALSE and run the model for a few periods to get what we accept as a limiting anplitude result, shown in Fig. 8 .

VI. DYN CODE LISTING

Owing to its length ( 31 pages), the DYN code 1 isting is not included in this report. However, anyone wanting a copy, may get it from the Los Alamos Scientific Laboratory, Group J-15 Group office. 


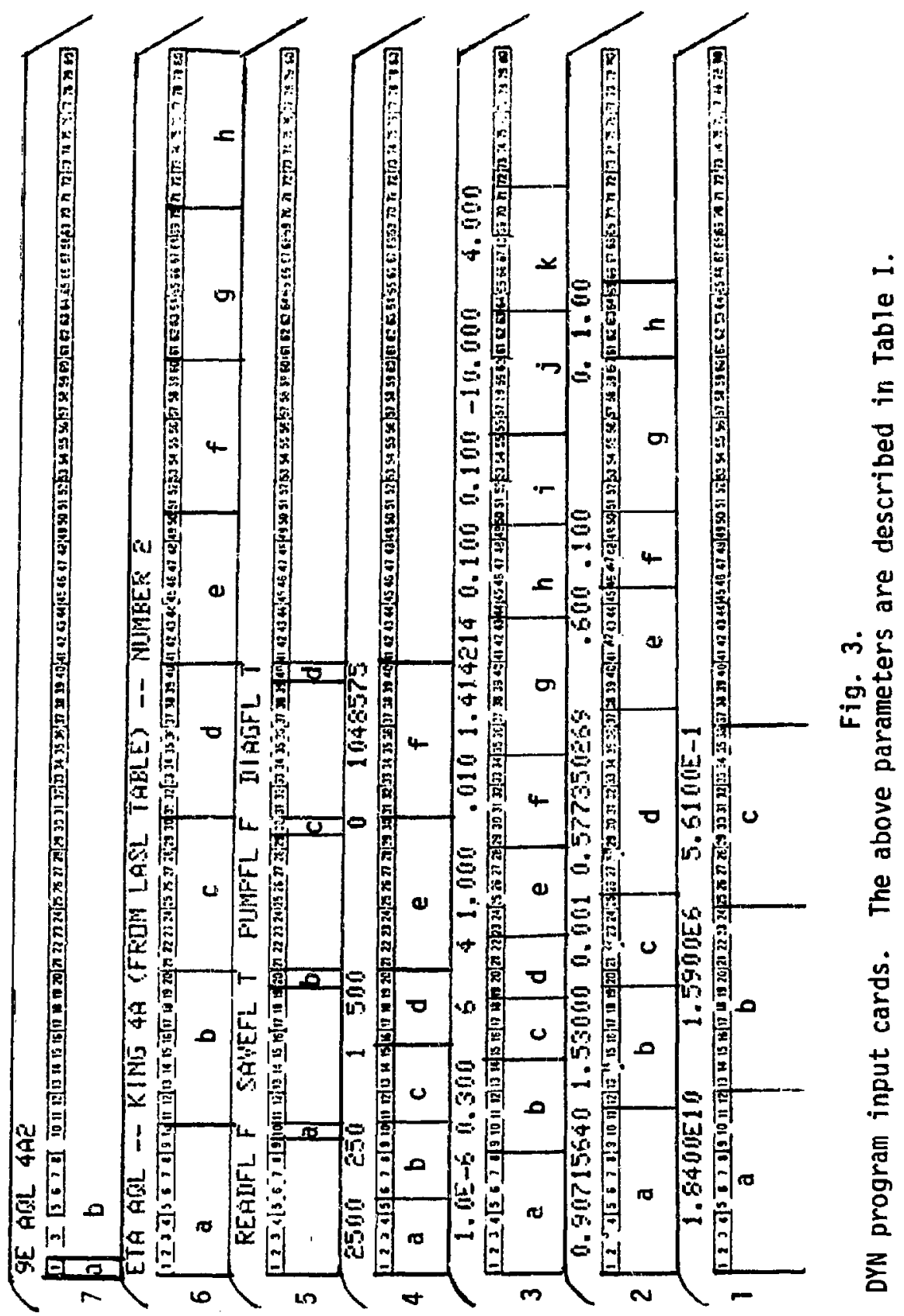


TABLE I

THE PARAMETERS THAT APPEAR ON THE DYN INPUT CARDS

(The names given are those within the program. The routine in which the parameter is used is given in the last column.)

\begin{tabular}{|c|c|c|c|c|}
\hline Card & Param. & Name & Description & Routine \\
\hline \multirow[t]{3}{*}{1} & a & NiT & Star mass $M_{s}$ & Static \\
\hline & b & LT & Luminosity $L_{T}$ & Static \\
\hline & c & IE & Effective temperature $T_{e}$ & Static \\
\hline \multirow[t]{8}{*}{2} & a & $X H$ & $\begin{array}{l}\text { Tabular helium abundance. See initial } \\
\text { routine }\end{array}$ & Initial \\
\hline & b & DMFAC & $\begin{array}{l}\left.\text { Nominal value of }\left(M_{s}-M_{i+1}\right) / M_{s}-M_{i}\right) \text { in } \\
\text { the Lagrangian region of the mesh. See } \\
\text { Eqs. (47) and }(48) \text {. }\end{array}$ & $\begin{array}{l}\text { Static, } \\
\text { Emfix }\end{array}$ \\
\hline & c & TAUO & Optical depth of outermost zone. & Static \\
\hline & $d$ & QO & $\begin{array}{l}\text { Value of the Hopf function that corresponds } \\
\text { to TAUO. }\end{array}$ & Static \\
\hline & e & DEVIATN & $\begin{array}{l}\text { Criterion for moving the active region } \\
\text { within the Lagrangian mesh. See Eq. (53). }\end{array}$ & Zonefix \\
\hline & $f$ & AEPS & $\begin{array}{l}\text { Centering feedback coefficient } \varepsilon_{A} \text {. See } \\
\text { Eq. }(52) \text {. }\end{array}$ & Emfix \\
\hline & g & AMX & $\left|A_{E}\right|$ dominance criterion $|\bar{A}|$. See Eq. (54). & Emfix \\
\hline & $h$ & Yex & Scaling exponent $\alpha$. See Eq. (56). & Emfix \\
\hline \multirow[t]{11}{*}{3} & a & EPS & Convergence criterion $\varepsilon$. See Fig. 2 . & DYN \\
\hline & b & TOL & Convergence criterion $\varepsilon^{\prime}$. See Fig. 2 . & DYN \\
\hline & c & ICMAX & $\begin{array}{l}\text { Solve iteration counter limit I s(max). } \\
\text { See Fig. } 2 \text {. }\end{array}$ & DYN \\
\hline & d & ICTSM & $\begin{array}{l}\text { Actempt counter limit } I_{A(\max x)^{\circ}} \\
\text { See Fig. } 2 \text {. }\end{array}$ & DYN \\
\hline & e & CQ & $\begin{array}{l}\text { Artificial viscosity ccefficient. } \\
\text { See Eq. }(16) \text {. }\end{array}$ & Solve \\
\hline & $f$ & CQCUT & $\begin{array}{l}\text { Artifiuial viscosity cutoff } G_{0} . \\
\text { See Eq. }(16) \text {. }\end{array}$ & Solve \\
\hline & $\mathbf{g}$ & DTFAC & Time step factor. & $\begin{array}{l}\text { Reset, } \\
\text { Accept }\end{array}$ \\
\hline & h & DTTOL & Temperature change tolerance. & Accept \\
\hline & $\mathbf{i}$ & DVTOL & Specific volume change tolerance. & Accept \\
\hline & j & UZERO & Initial velocity parameter. & Start \\
\hline & k & DTLIM & Limit on $\Delta t$. & Accept \\
\hline
\end{tabular}




\begin{tabular}{|c|c|c|c|c|}
\hline Card & Param. & Name & Description & Routine \\
\hline \multirow[t]{6}{*}{4} & a & NSTEP & $\begin{array}{l}\text { Limit on number of steps to calculate } \\
\text { (number of times through } C-D \text { loop in } \\
\text { Fig. 2). }\end{array}$ & DYN \\
\hline & b & NPRINT & $\begin{array}{l}\text { Modular print interval. Also controls } \\
\text { diagnostic plot interval. Zone-by-zone } \\
\text { prints and diagnostic plots arise when Hod } \\
\text { (ISTEP, NFRINT) }=0 \text {. ISTEP is the step } \\
\text { counter. See also 5d. }\end{array}$ & Accept \\
\hline & c & PLOTMOD & $\begin{array}{l}\text { Data for time plots (those indexed as } 1-5 \\
\text { in the plot list) are stored in 500- } \\
\text { element arrays that are entered when Mod } \\
\text { (ISTEP, PLOTMD) }=0 \text {. Plots are made when } \\
\text { the arrays are ful1. }\end{array}$ & Accept \\
\hline & d & SAVEMDD & $\begin{array}{l}\text { Modular dump interval. DYN dumps onto } \\
\text { the file MZSAVE whenever Mod (ISTEP, } \\
\text { SAVEMOD) }=0 \text {. SeE also 5b. }\end{array}$ & DYN \\
\hline & e & DMPSTEP & $\begin{array}{l}\text { Dump file step at which to begin problem } \\
\text { (currentiy a dummy variable). See also } 5 \text { a. }\end{array}$ & Restor \\
\hline & $f$ & PLTCTRL & $\begin{array}{l}\text { Integer whose bit pattern is analyzed } \\
\text { to determine which plots to process. }\end{array}$ & $\begin{array}{l}\text { Profils, } \\
\text { Ploter }\end{array}$ \\
\hline \multirow[t]{4}{*}{5} & $a$ & READFL & $\begin{array}{l}\text { Flag that controls decision to construct } \\
\text { a static model or to read the model from } \\
\text { the file MZSAVE. See Fig. } 2 \text {. }\end{array}$ & Start \\
\hline & b & SAVEFL & $\begin{array}{l}\text { Flag that controls decision to make a } \\
\text { dump on the save file MZSAVE. See Fig. } 2 \text {. }\end{array}$ & DYN \\
\hline & c & PUMPFL & $\begin{array}{l}\text { When this flag is set to TRUE, velocities } \\
\text { are increased } 25 \% \text { at every kinetic energy } \\
\text { maximum. Thus one may "pump" a model } \\
\text { rapidly to limiting amplitude. }\end{array}$ & Accept \\
\hline & d & DIAGFL & $\begin{array}{l}\text { Flag that controls whether diagnostic } \\
\text { plots are made. It overrides the selec- } \\
\text { tion implied by the PLTCTRL variable, } \\
\text { parameter } 4 \mathrm{f} \text {, but does not interfere } \\
\text { with the time plots. Time plots are } \\
\text { avoided by turning the lower li bits of } \\
\text { PLTCTRL "off". }\end{array}$ & Accept \\
\hline 6 & $a-h$ & $x$ & Listing title card. & Initial \\
\hline \multirow[t]{2}{*}{7} & a & NCHMONA & $\begin{array}{l}\text { Number of characters ( } 9 \text { or fewer) in the } \\
\text { model name. See } 7 \mathrm{~b} \text {. }\end{array}$ & Start \\
\hline & b & MODEL & Model name that appears on the plots. & Start \\
\hline
\end{tabular}




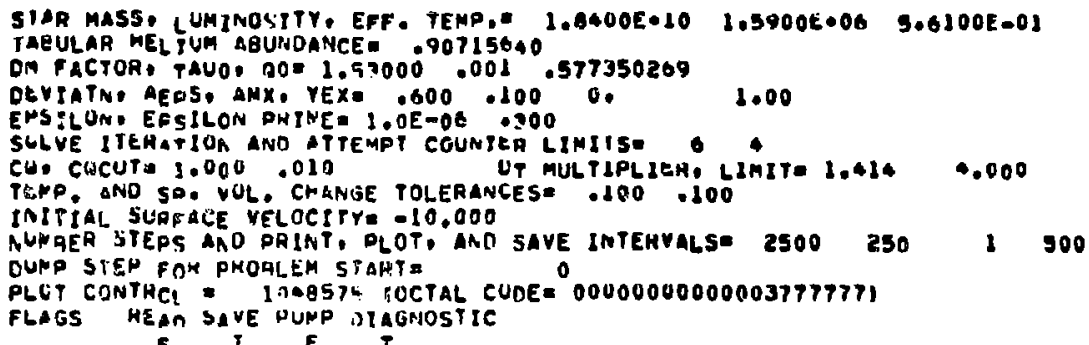

Fig. 4.

Listing of program output heading. The PLTCTRL parameter (4f of Fig. 3) is listed both in integer and octal code. The letter is included for simple dissection of the integer PLTCTRL into its bit pattern. 


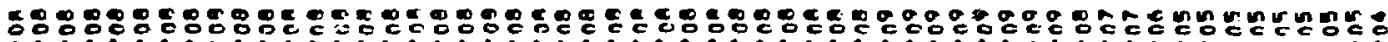

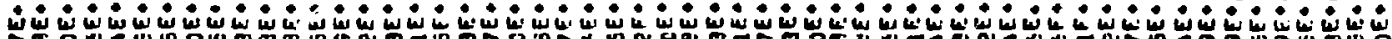

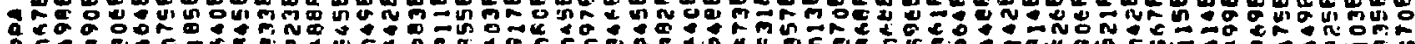
géñ

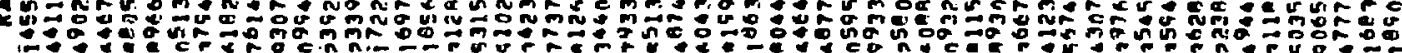

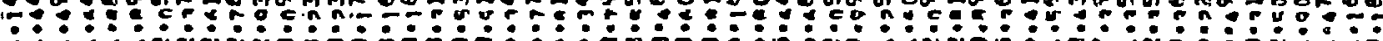

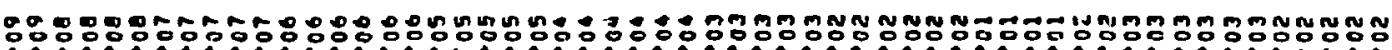

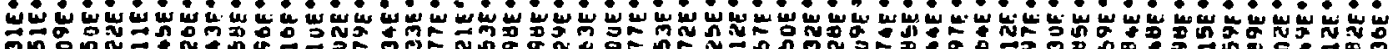

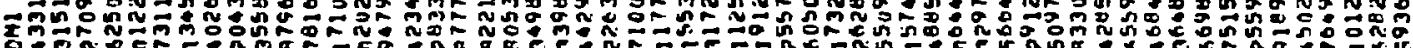

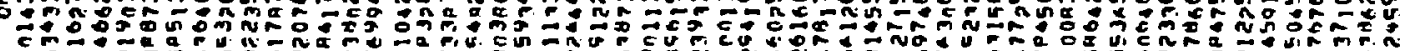

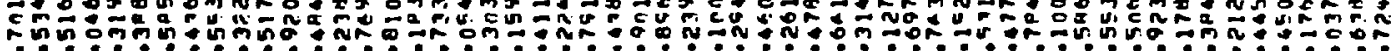
m:

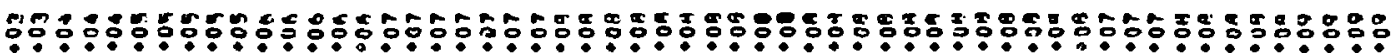

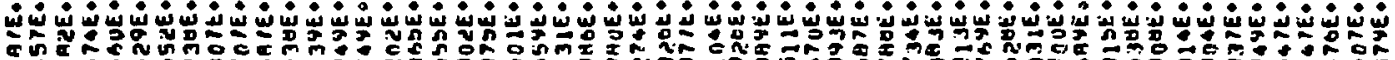
$>$ Non

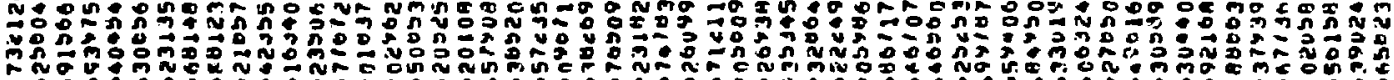
:.0:

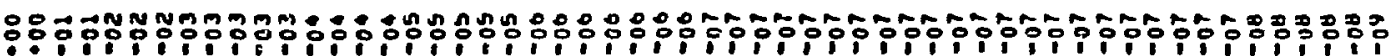

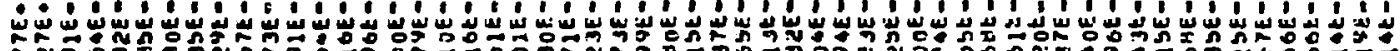

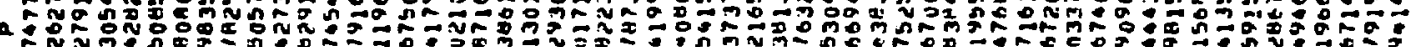

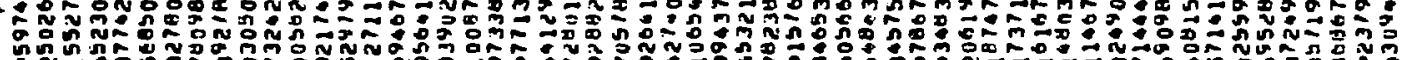

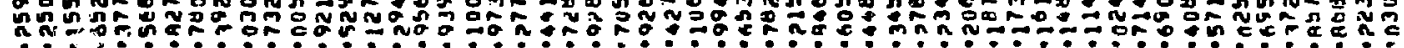

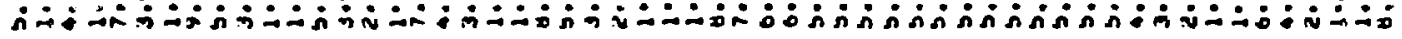

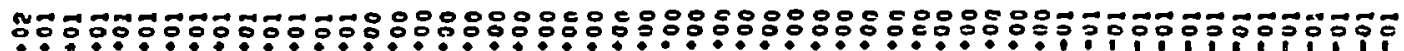

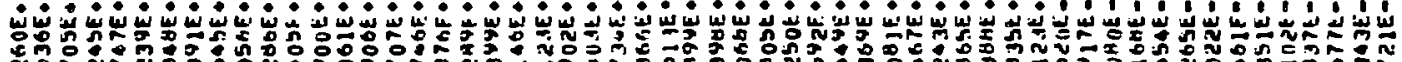

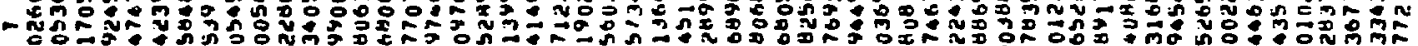

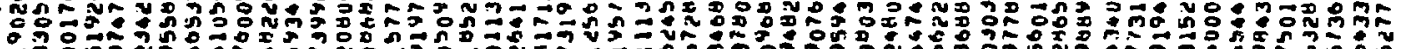

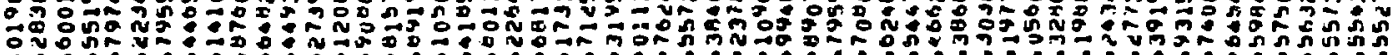

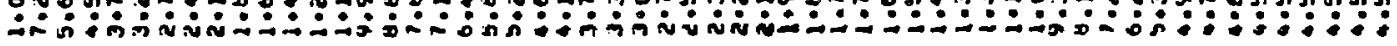

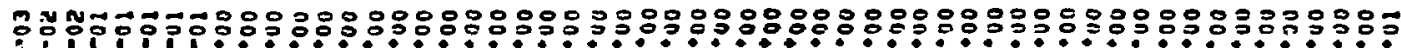

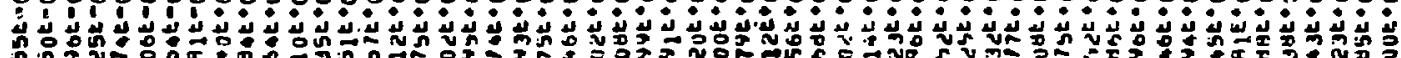

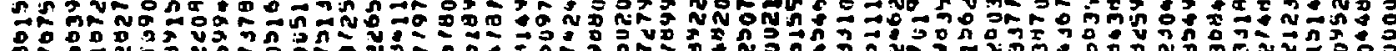

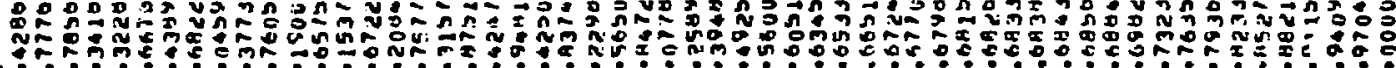

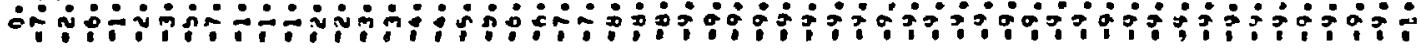

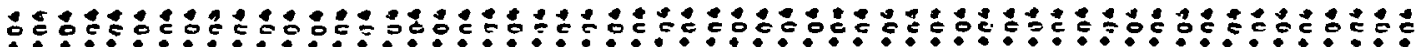

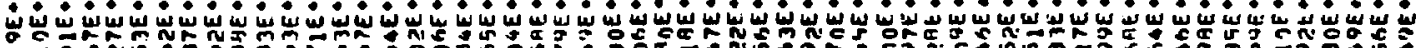

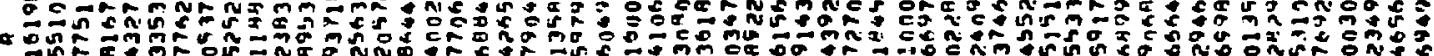

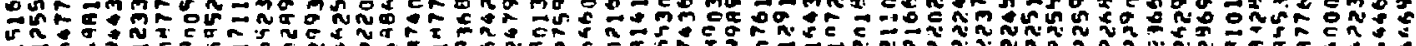

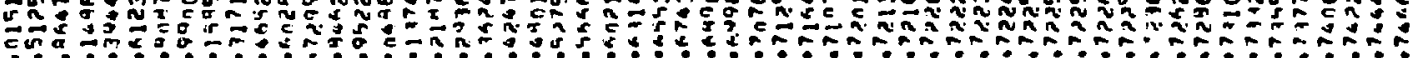

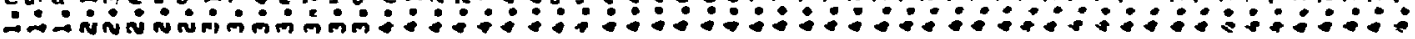

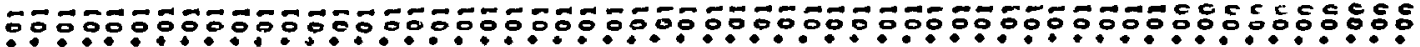

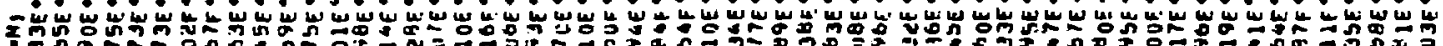

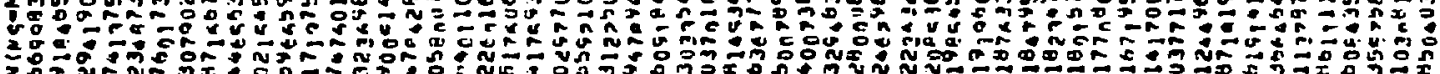

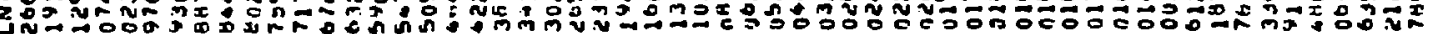
ก ค

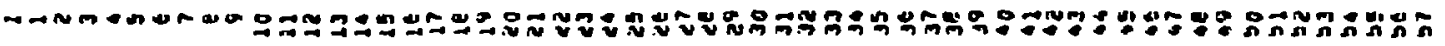




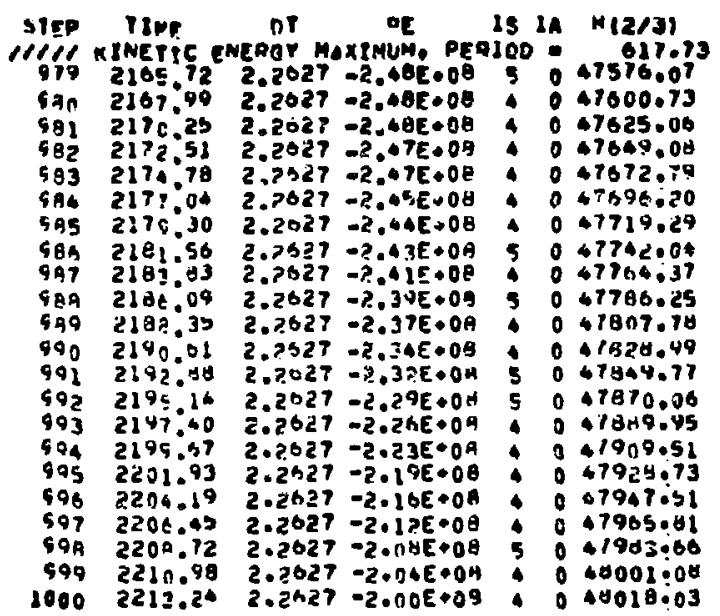

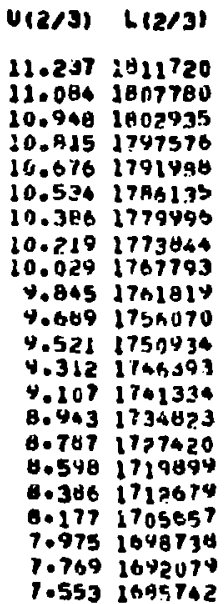

EekK

11 JJO.d 22/3 27 I PMASE

$.57721 \quad 2.209 E \cdot 0010.0996-7.86 E \cdot 04$ .57672 2.2B.3E+08 $10.1083-8.11 E+n 4$ -57617 2.275E 0 $10.1171-9.36 E \cdot 94$

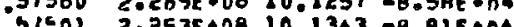
- STsar 2.

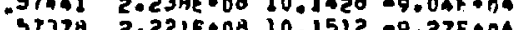
-57315

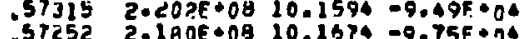
57189 2.15AE+08 $10.1754=0.08 E+n E$

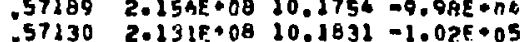
$.5730201316 .0820 .1831=1.02 E+05$

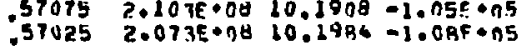
.50570 2.0A?F-n\& $10.2060-1.10 \mathrm{f} \cdot 05$ $.564072 .00 B F .0810 .2136-1.13 F \cdot 05$ $.50439 \quad 1.073 E \cdot 38 \quad 10.2 E 10-1 \cdot 16 E \cdot n 5$ $.367691 .934 E \cdot 0010.2291$ - 1.1850 .05

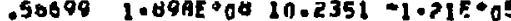
$.566301 .06 A E \cdot 00$ 1n.2419-1.2.3E 05 .56563 1.61AE.06 10.24A5 - $1.26 E$ * O5 $.56498 \quad 1.773 E^{\circ} 0610.2550-1.26 F \cdot 05$ .56436

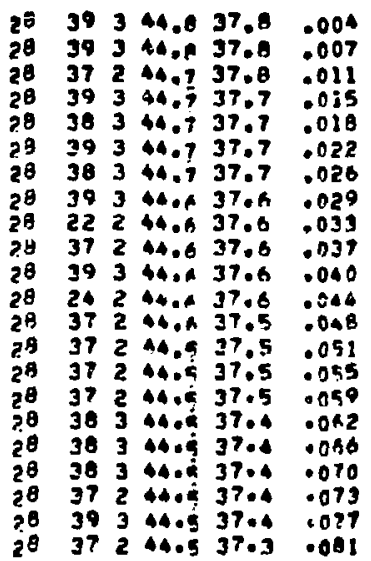

Fig. 6.

Partial listing immediately preceding the variable 1 isting at step 1000 . Kinetic energy maxima are flagged on the output listing. Here we see that the program calculates a period of 7.15 days from kinetic energy maximum data. The data under the heading PE are a normalized combination of gravitational and internal energies. $I_{S}$ and $I_{A}$ are the solve and attempt counters indicated in Fig. 2. The " $2 / 3$ " after the $R, u$, and $T$ headings indicates that the listed radii, velocities, and temperatures are measured at optical depth $\tau=2 / 3 . \quad M(T=1)$ is the mass depth of the point at which $T=10000 \mathrm{~K}$ (the location of the hydrogen ionization front). ECHK is a normalized energy check. $I 1$ is the lower edge of the active region. JJO and JJl are the indexes of the zone controlling the timing and of the corresponding parameter $(1 \leftrightarrow u, 2 \leftrightarrow T, 3 \leftrightarrow R) . \quad Z 2 / 3$ and $Z t=2$ are the locations of optical depth $\tau=2 / 3$ and of the hydrogen ionization front (where $T=10000 \mathrm{~K}$ ). 


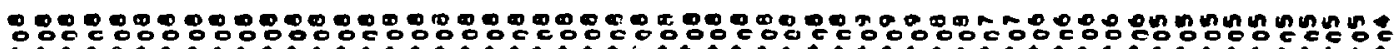
Q

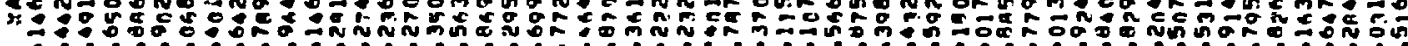

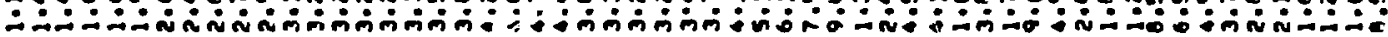

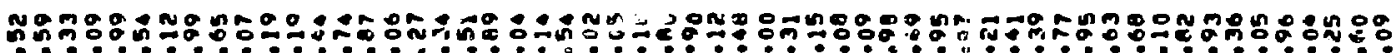
w

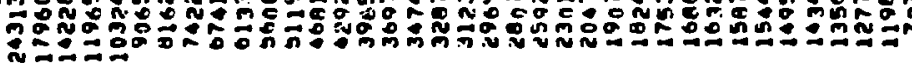

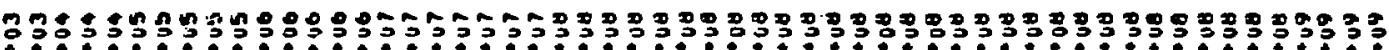

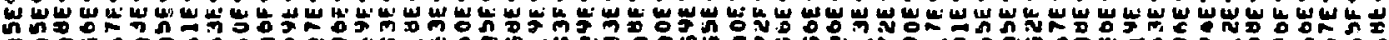

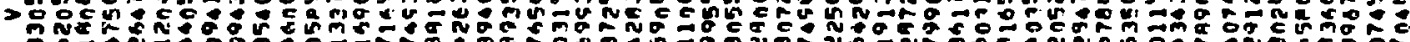

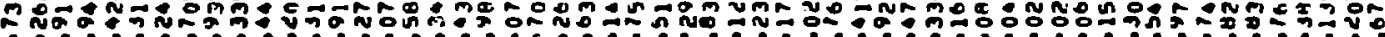

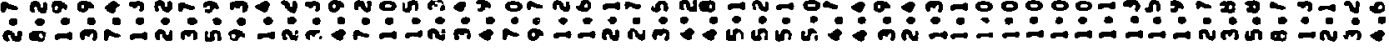

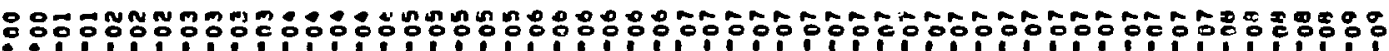

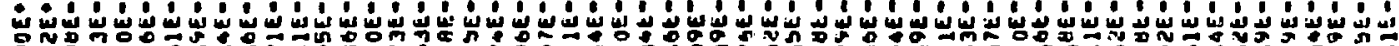

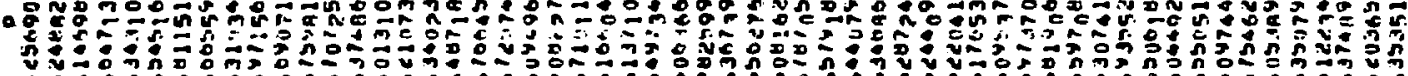
í:

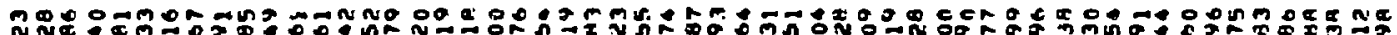

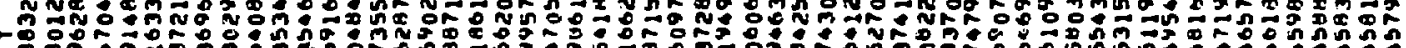

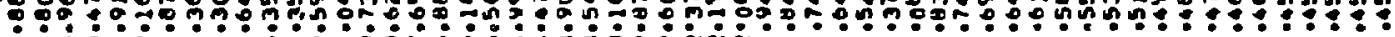

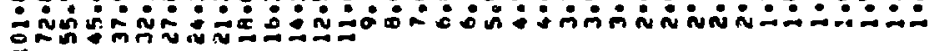

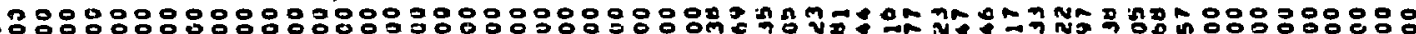

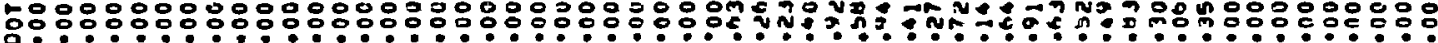

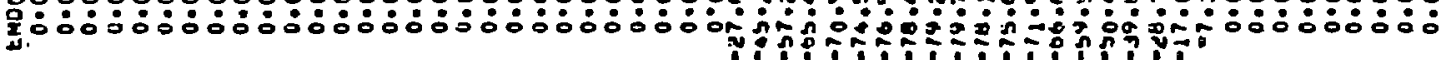

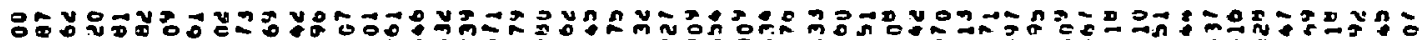


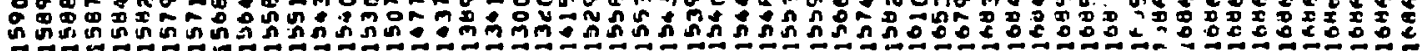

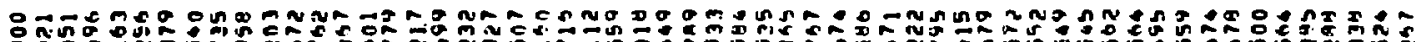

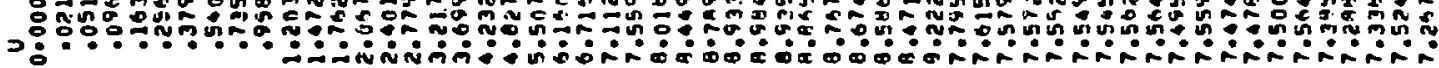

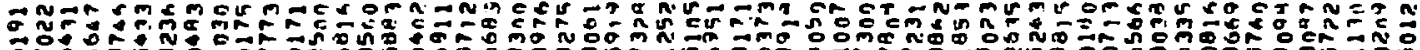

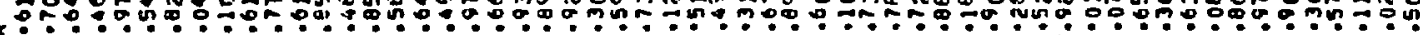
-

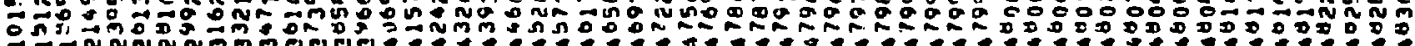

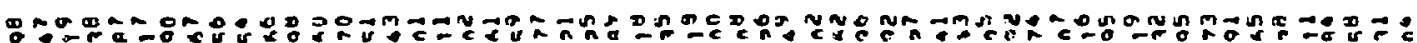
: ฟ

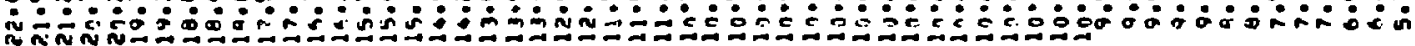

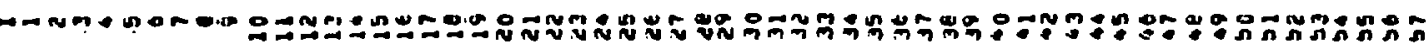

is 

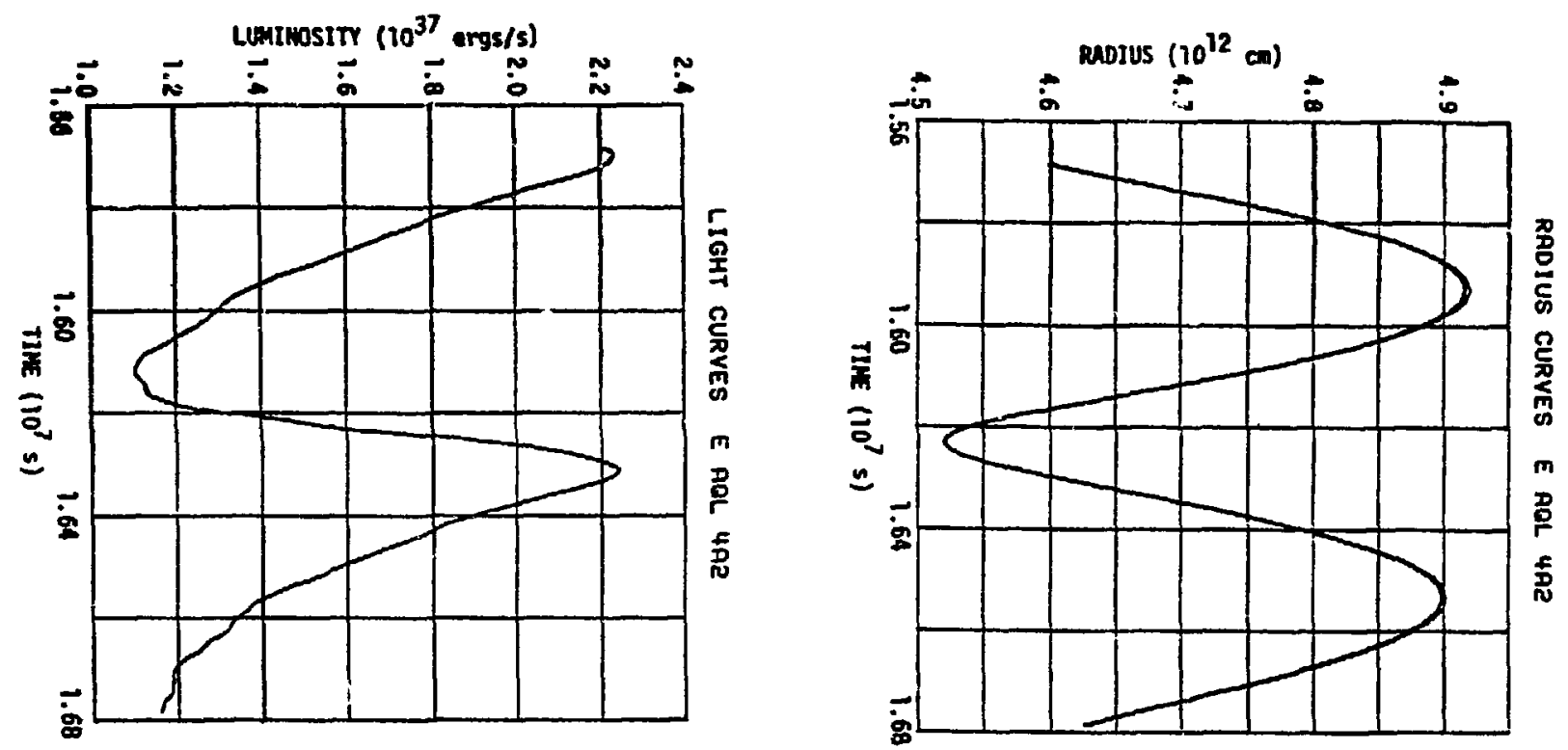

증웅

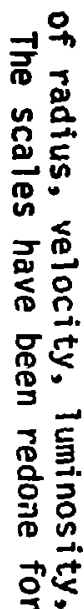

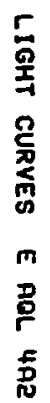

응

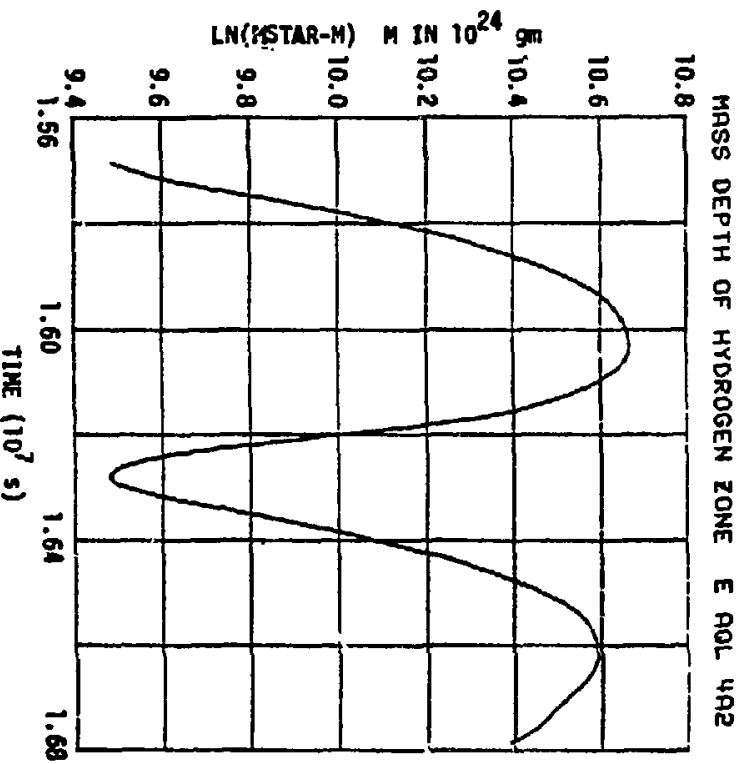

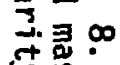

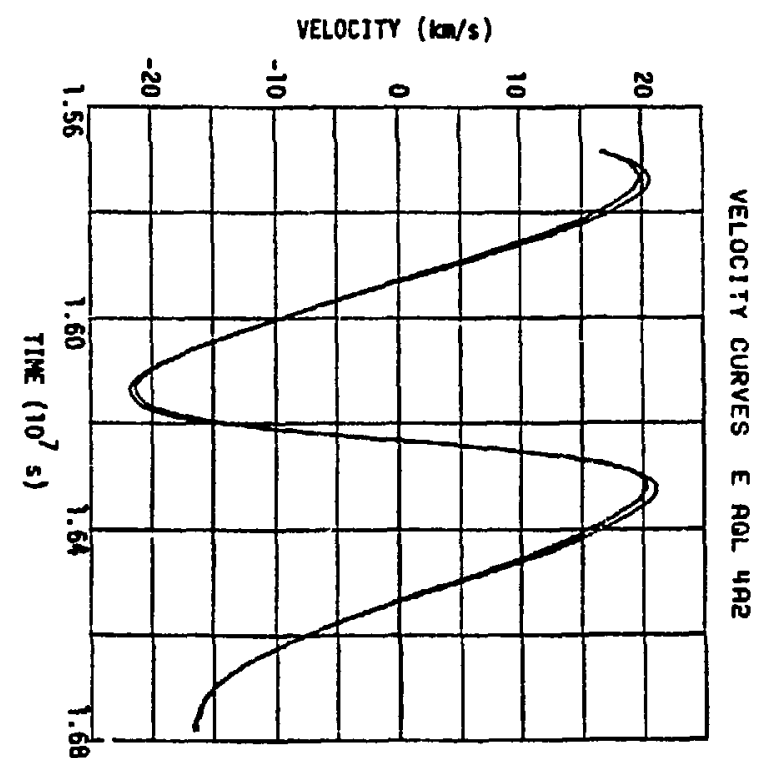




\section{REFERENCES}

1. C. F. Keller and J. P. Mutschlecner, "Hydrodynamic Envelopes of Classical Cepheid Variables," Ap. J. 167, 127 (1971).

2. G. S. Fraley, "Supernovae Explosions Induced by Pair-Production Instability," Astrophys. and Space Sci. 2, 96 (1968).

3. R. D. Richtmyer and K. W. Morton, Finite Difference Methods for Initial Value Problems, 2nd Ed. (Interscience, New York, 1967).

4. D. Mihalas, Stellär Atmospheres (Freeman, San Francisco, 1970).

5. J. W. Pel, "A Photometric Study of Cepheids in the Sosthern Milky Way," Ph. D. Thesis, Ryksuniversiteit, Leiden, $19 ; 6$.

6. C. Whitney, "The Radii of $\delta$ Cephei and $n$ Aquilae," Ap. J. 121, 682 (1955).

7. J. B. Oke, "An Analysis of the Absolute Energy Distribution in the Spectrum of Eta Aquilae," Ap. J. 133, 90 (1961).

8. E. G. Schmidt, "The Temperature Scale of $F$ and $G$ Stars. I. Hydrogen Line Profiles," Ap. J. 174, 595 (1972).

9. R. F. Christy, "Energy Transport in the Hydrogen Ionization Zone of Giant Stars," Ap. J. 136, 887(1962).

10. D. S. King, J. P. Cox, D. D. Eilers, and W. R. Davey, "Nonlinear Cepheid Pulsation Calculations and Comparisons with Linear Theory," Ap. J. 182, 859 (1973).

11. J. P. Cox, "Pulsating Stars," Rep. Prog. Phys. 37, 563 (1974). 